In Their Own Best Interest 



\section{In Their Own Best Interest}

A History of the U.S. Effort to Improve Latin Americans

LARS SCHOULTZ

II Harvard University Press

Cambridge, Massachusetts \& London, England / 2018 
Copyright ( 2018 by the President and Fellows of Harvard College

All rights reserved

Printed in the United States of America

First printing

Library of Congress Cataloging-in-Publication Data

Names: Schoultz, Lars, author.

Title: In their own best interest : a history of the U.S. effort to improve Latin Americans /

Lars Schoultz.

Description: Cambridge, Massachusetts : Harvard University Press, 20I8. | Includes

bibliographical references and index.

Identifiers: LCCN 201706I473 | ISBN 9780674984I4I (alk. paper)

Subjects: LCSH: United States—Foreign relations-Latin America. | Latin America—Foreign

relations_-United States. | United States—Foreign relations—Moral and ethical aspects. |

United States—Foreign relations_-Philosophy. | Politicians_-United States—Attitudes. |

Economic assistance, American-Latin America-History. | Progressivism (United States politics)

Classification: LCC FI418 . S395 2018 | DDC 327.7308—dc23 LC record available at

https://lccn.loc.gov/2017061473

Jacket design: Jill Breitbarth

Jacket image: USAID logo (detail) 
For Jane 
